\title{
DETERMINATION OF OPACITY DATA BASES FOR TIO AND $\mathrm{H}_{2} \mathrm{O}$
}

\author{
S.R. LANGHOFF, D.W. SCHWENKE AND H. PARTRIDGE \\ Mail Stop 230-3 \\ NASA Ames Research Center \\ Moffett Field, CA 94035, USA
}

\begin{abstract}
Current $a b$ initio methods for determining potential energy surfaces are discussed in relation to the $\mathrm{TiO}$ and $\mathrm{H}_{2} \mathrm{O}$ molecules, both of which make important contributions to the opacity of M-type stars. For the $\mathrm{TiO}$ molecule we discuss the determination of the radiative lifetimes of the excited states and band oscillator strengths for both the triplet and singlet band systems. While the theoretical radiative lifetimes for $\mathrm{TiO}$ agree well with recent measurements, the band oscillator strengths differ significantly from those currently employed in opacity calculations. For the $\mathrm{H}_{2} \mathrm{O}$ molecule we discuss the current results for the ground state potential energy and dipole moment surfaces generated at NASA Ames. We show that it is necessary to account for such effects as core-valence correlation to generate a potential energy surface of near spectroscopic accuracy. The current status of our effort to establish opacity data bases for both $\mathrm{TiO}$ and $\mathrm{H}_{2} \mathrm{O}$ is described.
\end{abstract}

\section{Introduction}

The $\mathrm{TiO}$ and $\mathrm{H}_{2} \mathrm{O}$ molecules play a dominant role in the opacity of M-type stars throughout the visible and near infrared (Merrill, Deutsch \& Keenan 1962). Thus there has been considerable effort to determine accurate opacity data bases for these two molecules. Quantum mechanical calculations are essential to this effort, because it is not possible to account for the tens of millions of lines that are present at stellar temperatures by experimental means. Theory offers the possibility of obtaining complete opacity data bases if the potential energy and transition moment (or dipole moment) surfaces can be determined with sufficient accuracy. In this paper we de- 
scribe the effort at NASA Ames to obtain quantitative opacity data bases for the $\mathrm{TiO}$ and $\mathrm{H}_{2} \mathrm{O}$ molecules.

The currently accepted constants and band oscillator strengths for all of the important transitions in $\mathrm{TiO}$ have been summarized recently by Jørgensen (1994). He has used this data to construct an opacity list for $\mathrm{TiO}$ that is in current use in stellar modeling. His recommended oscillator strengths differ considerably from the earlier work of Collins (1975), but are in reasonable accord with the work of Brett (1990). With the exception of the $\delta$ band system, all of the oscillator strengths determined in this work are less (and in several cases significantly less) than Jørgensen's best estimates. However, our calculated radiative lifetimes agree well with those recently measured under collision-free conditions in a supersonic molecular beam (Hedgecock, Naulin \& Costes 1995). In addition, we calculate a lifetime for the $E^{3} \Pi$ state of over $4000 \mathrm{nsec}$, in agreement with the lower limit of $\tau>$ $2000 \mathrm{nsec}$, but in disagreement with an earlier determination of $770 \pm 40 \mathrm{nsec}$ by Simard \& Hackett (1991).

Considerable effort has been devoted to constructing a globally correct potential energy surface for the ${ }^{1} A_{1}$ ground electronic state of the $\mathrm{H}_{2} \mathrm{O}$ molecule. Quantum mechanical calculations themselves are not capable of generating a potential energy surface (PES) of spectroscopic accuracy. The procedure that is followed is to determine the energy at a discrete set of geometries and then fit these to analytical form. The form of the potential is then adjusted empirically to match known line position, e.g. those in the HITRAN data base (Rothman et al. 1995). For this procedure to give a quantitative PES capable of describing the $\mathrm{H}_{2} \mathrm{O}$ molecule to near its dissociation limit, it is necessary to have $a b$ initio data at all representative points on the surface, to have an accurate analytical fit to the discrete energy points, and to have accounted for all differential energy effects, such as core-valence (oxygen $1 s$ ) electron correlation that affects the shape of the potential. We outline the construction of a PES for water that describes all the energy levels $(J \leq 5)$ in the HITRAN data base with a root-mean square deviation of about $0.044 \mathrm{~cm}^{-1}$. We believe that our empirically corrected PES is of quantitative accuracy at all energies up to $40,000 \mathrm{~cm}^{-1}$. In this paper we describe the construction of this surface and the subsequent calculations to generate an opacity data base for $\mathrm{H}_{2} \mathrm{O}$.

\section{TiO molecule}

The principal goal of the theoretical work (Langhoff 1996) on TiO was to determine $g f$ values for all absorption lines that would contribute to the opacity of cool evolved stars (temperatures on the order of $3000 \mathrm{~K}$ ). This in turn requires reliable transition moment functions (TMFs) for 
all dipole-allowed transitions out of the $X^{3} \Delta$ ground and low-lying $a^{1} \Delta$ states. To obtain these data we have performed complete-active-space selfconsistent-field (CASSCF) followed by internally-contracted multireference configuration-interaction (IC-MRCI) calculations. These calculations performed in reasonably complete one-particle basis sets account for a significant fraction of the valence correlation energy and give potential energy curves in rather good agreement with Klein-Dunham potentials (Langhoff 1996). The greatest difficulty in the theoretical calculations is following the potentials to larger internuclear distances ( $r$ values), due to the presence of other weakly bound states that lie below the excited states that are the lowest states in the Franck-Condon region. Nevertheless, TMFs can be determined over a sufficiently large region of $r$ values to give accurate radiative lifetimes for at least the lower vibrational levels of the excited states, as well as accurate opacity data, because all of the TMFs fall off substantially in magnitude as $r$ increases.

The calculated radiative lifetimes of the excited states are compared with the available experimental data in Table 1 . The recent lifetime data of Hedgecock et al. (1995) obtained under collision free conditions in a supersonic expansion should be the most reliable. The lifetimes computed in an earlier theoretical study by Schamps, Sennesal \& Carette (1992) at the single-reference CI level are also included in Table 1. The IC-MRCI lifetimes from the present study are expected to be accurate to about $20 \%$ (except possibly for the weak $\epsilon$ system). The agreement with the recent lifetime data of Hedgecock et al. (1995) is excellent. Except for the upper state of the $\delta$ system $\left(b^{1} \Pi\right.$ state), the present theoretical lifetimes agree with those of Schamps et al. (1992) to better than a factor of two.

The largest discrepancies with previous work are for the $E^{3} \Pi-X^{3} \Delta$ $\epsilon$ and $b^{1} \Pi-a^{1} \Delta \delta$ systems. For the $\epsilon$ system we obtain almost a factor of two increase in the lifetime between the CASSCF and IC-MRCI levels. Our IC-MRCI lifetime of $4259 \mathrm{nsec}$ for $v=0$ is consistent with the lower bound of $>2000$ nsec determined by Hedgecock et al. (1995), but is significantly longer than the determination of $770 \pm 40 \mathrm{nsec}$ of Simard \& Hackett (1991). Also, considering the sensitivity of the lifetime to the level of electron correlation, it is not surprising that it is also longer than the earlier value of 2300 nsec calculated by Schamps et al. (1992). Since we believe the theoretical value is accurate to within a factor of two, we suspect that the earlier experimental measurement may not have adequately accounted for diffusion effects. Our IC-MRCI lifetime for the $b^{1} \Pi$ state of $119.7 \mathrm{nsec}$ (for $v=0$ ) is significantly shorter than the earlier theoretical value of $455 \mathrm{nsec}$ obtained by Schamps et al. (1992). This can be traced to the fact that we obtain much larger transition probabilities for the $b^{1} \Pi-a^{1} \Delta \delta$ band system. An experimental determination of the lifetime for this state would be 
TABLE 1. Lifetimes (nsec) of the triplet and singlet states of $\mathrm{TiO}$

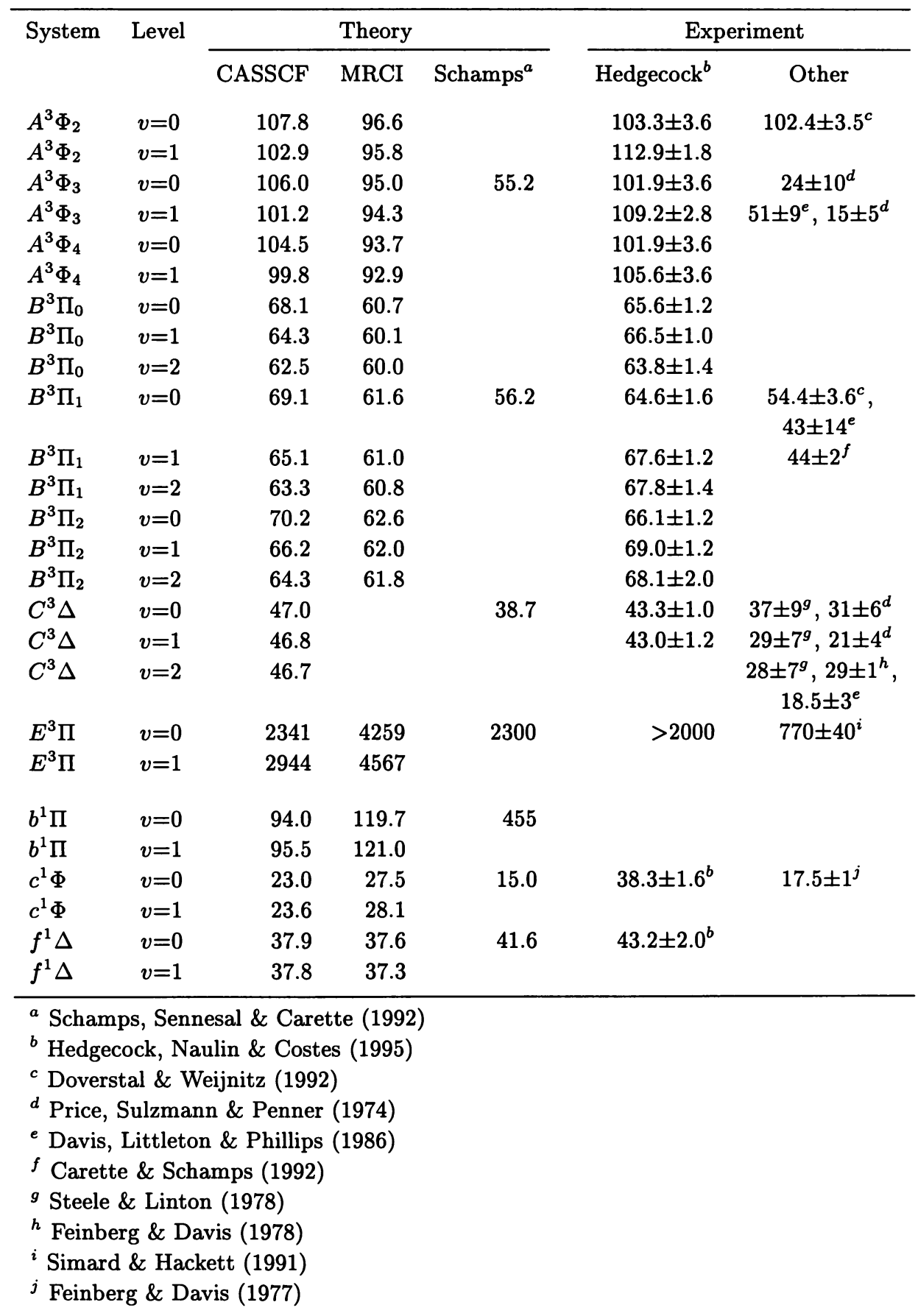


TABLE 2. Electronic oscillator strengths $f_{e l}\left(\nu_{00}\right)$ for the band systems of $\mathrm{TiO}$

\begin{tabular}{lcrrrr}
\hline Transition & $\nu_{00}\left(\mathrm{~cm}^{-1}\right)$ & \multicolumn{5}{c}{$f_{e l}\left(\nu_{00}\right)$} \\
\cline { 3 - 6 } & & This work $^{a}$ & Jørgenson $^{b}$ & Brett $^{c}$ & Collins $^{d}$ \\
$\alpha\left(C^{3} \Delta-X^{3} \Delta\right)$ & 19339.0 & 0.105 & 0.17 & & 0.12 \\
$\beta\left(c^{1} \Phi-a^{1} \Delta\right)$ & 17840.6 & 0.176 & 0.28 & & 0.006 \\
$\gamma^{\prime}\left(B^{3} \Pi-X^{3} \Delta\right)$ & 16147.0 & 0.108 & 0.14 & & 0.25 \\
$\gamma\left(A^{3} \Phi-X^{3} \Delta\right)$ & 14092.9 & 0.092 & 0.15 & $0.22 \pm 0.04$ & 0.31 \\
$\epsilon\left(E^{3} \Pi-X^{3} \Delta\right)$ & 11893.9 & 0.002 & 0.014 & $0.006 \pm 0.001$ & 0.16 \\
$\delta\left(b^{1} \Pi-a^{1} \Delta\right)$ & 11273.3 & 0.096 & 0.048 & 0.05 & 0.006 \\
$\phi\left(b^{1} \Pi-d^{1} \Sigma^{+}\right)$ & 9054.0 & 0.018 & 0.052 & $0.05 \pm 0.01$ & 0.006 \\
\hline${ }^{a}$ MRCI values. & & & & & \\
${ }^{b}$ Jørgensen recommended values (1994). & & & & \\
${ }^{c}$ Brett (1990). & & & & & \\
${ }^{d}$ Collins (1975). & & & & &
\end{tabular}

highly desirable.

In Table 2 we compare our calculated $f_{e l}\left(\nu_{00}\right)$ values for selected transitions with the recommendations of Jørgensen (1994) and with the earlier values reported by Brett (1990) and Collins (1975). Except for the $\delta$ bands, the recommended values of Jørgensen (1994) are larger and in some cases, e.g. the $\epsilon$ and $\phi$ bands, considerably larger than the current theoretical values. We believe that the theoretical oscillator strengths, at least for the stronger bands, are accurate to $20 \%$. It would thus be very desirable to generate opacity data based on the IC-MRCI TMFs. We are presently working on generating a complete data base of transition probabilities for all of the band systems studied in this work. We find that to achieve completeness in the opacity for temperatures of $3000 \mathrm{~K}$, that it is necessary to include levels with very high $v$ and $J$ values. For example, the $A^{3} \Phi-X^{3} \Delta \gamma$ band system shown in Fig. 1 for a temperature of $3000 \mathrm{~K}$ contains 718334 lines involving $J$ up to 238 (see figure caption).

\section{3. $\mathrm{H}_{2} \mathrm{O}$ molecule}

The $\mathrm{H}_{2} \mathrm{O}$ molecule also plays an important role in determining the opacity of oxygen-rich cool evolved stars. Thus a considerable effort has gone into developing opacity data bases for $\mathrm{H}_{2} \mathrm{O}$ (Jørgensen \& Jensen 1993; Polyansky et al. 1996; Viti \& Tennyson 1996). In this section we describe the work ongoing at Ames (Partridge \& Schwenke 1996) to provide a complete line list of near spectroscopic accuracy for energies below about $30,000 \mathrm{~cm}^{-1}$ 


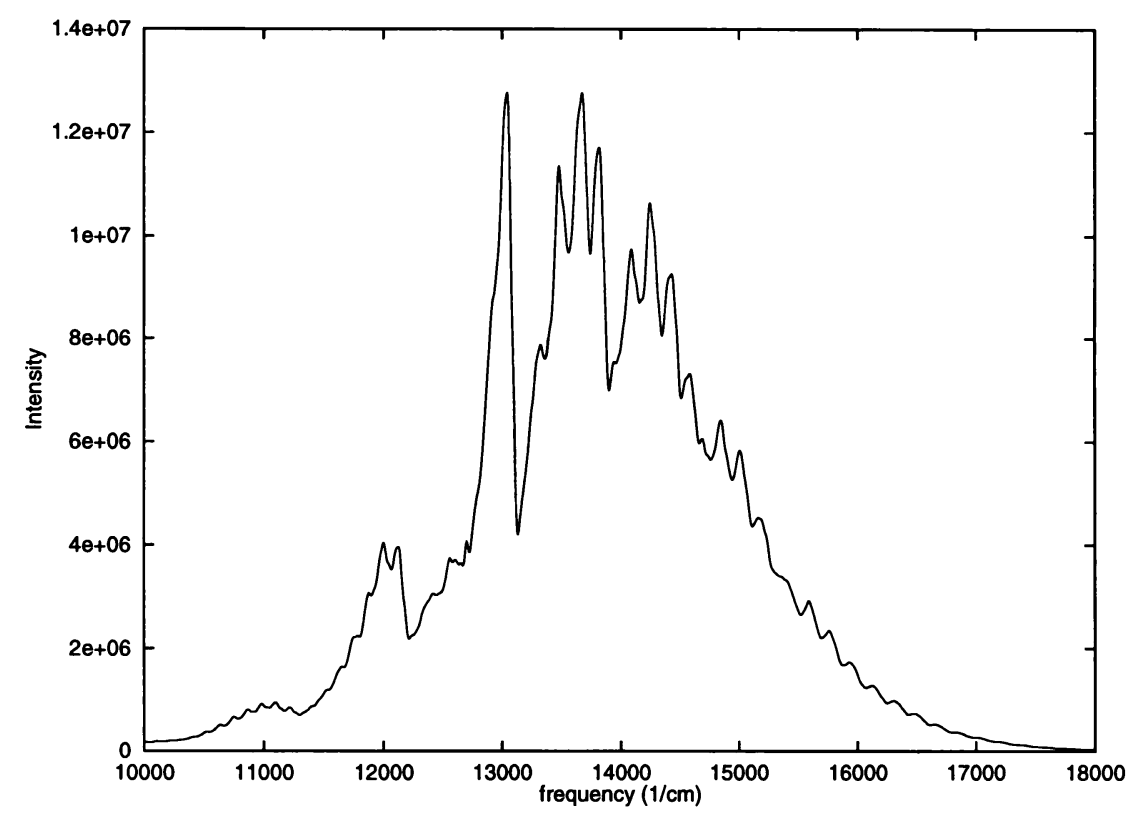

Figure 1. The $A^{3} \Phi-X^{3} \Delta \delta$ band system of TiO. The spectrum contains all of the lines (FWHM of $10 \mathrm{~cm}^{-1}$ ) with populations $\times$ (dipole moment matrix element) ${ }^{2}$ at $3000 \mathrm{~K}$ greater than $1 \times 10^{-8}$ (a total of 718334 lines). The intensity has arbitrary units.

that will be quantitative for very high temperatures (4000-5000 K).

The first step in generating a globally accurate PES for $\mathrm{H}_{2} \mathrm{O}$ molecule is to determine the energy for representative geometrical arrangements of the atoms with energies below $40,000 \mathrm{~cm}^{-1}$. Our theoretical potential was determined in $C_{s}$ symmetry at 771 geometries using the correlation-consistent polarized quintuple-zeta basis sets (Dunning 1989; Kendall, Dunning \& Harrison 1992) augmented with diffuse $s, p$, and $d$ functions on oxygen and $s$ and $p$ functions on hydrogen. The molecular orbitals were determined at the CASSCF level with a $\left(6 a^{\prime} 2 a^{\prime \prime}\right)$ active space and correlating the eight valence electrons of $\mathrm{H}_{2} \mathrm{O}$. Electron correlation was then included by performing an IC-MRCI calculation and accounting for higher excitations with a multireference quadruples correction $(+Q)$. This potential is denoted as $\mathrm{V}^{5 z}$. Based on a series of larger calibration calculations it was discovered that the largest differential effect on the PES missing in the above calculation was the neglect of core (oxygen $1 s$ ) correlation. Thus we added a correction for core correlation as the difference in energies between two calculations with eight and ten electrons correlated using a basis set including core correlation functions derived from a correlation-consistent polarized 
quadruple-zeta basis. Electron correlation was included using the averaged coupled pair functional (ACPF) method to avoid problems with size inconsistency. This core correction is denoted $\Delta V^{\text {core }}$. As an estimate of the effect of further improvements in the one-particle basis sets, we also computed the difference between the quadruple-zeta and quintuple-zeta valence calculations. This correction is denoted $\Delta V^{\text {basis }}$.

The next step in our procedure is to develop an analytical representation of the PES that accurately represents the energies and has the correct asymptotic form. This is done by representing the $\mathrm{V}^{5 z}$ potential as the sum of two- and three-body terms. The $\Delta V^{\text {core }}$ and $\Delta V^{\text {basis }}$ corrections are fit using only three-body terms. It is necessary to obtain a very accurate fit in order for the empirical corrections described below to give good results.

The $V^{5 z}$ PES even with core $\left(\Delta V^{\text {core }}\right)$ and basis set $\left(\Delta V^{\text {basis }}\right)$ corrections is not sufficiently accurate to give line positions with near spectroscopic $\left(0.1 \mathrm{~cm}^{-1}\right)$ accuracy. In addition to the effects of incompleteness in both the one- and $n$ particle expansions, we have neglected corrections such as relativity, mass polarization, and diagonal adiabatic modifications to the Born-Oppenheimer approximation. We attempt to include all of these effects by generating an empirical PES of the form:

$$
\begin{array}{r}
V^{\text {emp }}\left(r_{1}, r_{2}, \theta\right)=c^{5 z} V^{5 z}\left(r_{1}, r_{2}, \theta\right)+c^{\text {core }} \Delta V^{\text {core }}\left(r_{1}, r_{2}, \theta\right) \\
+c^{\text {basis }} \Delta V^{\text {basis }}\left(r_{1}, r_{2}, \theta\right)+\Delta V^{\text {rest }}\left(r_{1}, r_{2}, \theta\right),
\end{array}
$$

with $\Delta V^{\text {rest }}$ represented by three-body terms. Our final PES had 21 parameters, which were chosen by fitting to lines in the HITRAN data base (Rothman et al. 1995) with $J \leq 5$. The root-mean-square (rms) error is only $0.044 \mathrm{~cm}^{-1}$. For this PES, the accuracy remains good for higher $J$ as well. Comparing to all HITRAN lines gives an rms error of $0.125 \mathrm{~cm}^{-1}$. This number is deceptively high, because $69 \%$ of the lines $(18,806$ lines) have errors less than $0.05 \mathrm{~cm}^{-1}$ and the outliers are most likely missassigned lines.

Using the above PES and an analytical representation of our dipole moment surface, we obtained the transition intensities and energies using the algorithm described by Schwenke (1996). The vibrational wave functions were represented by linear combinations of analytical basis functions with the expansion coefficients determined by the variational principal. The exact kinetic energy operator is used in all calculations. The variational calculations can be made sufficiently complete even for high $J$ values, so that the limitations in the transition intensities and energies derive almost completely from the PES. As the energy increases the density of states increases dramatically and the individual levels become a mixture of normal modes making it difficult to give specific assignments to the levels. Although the 


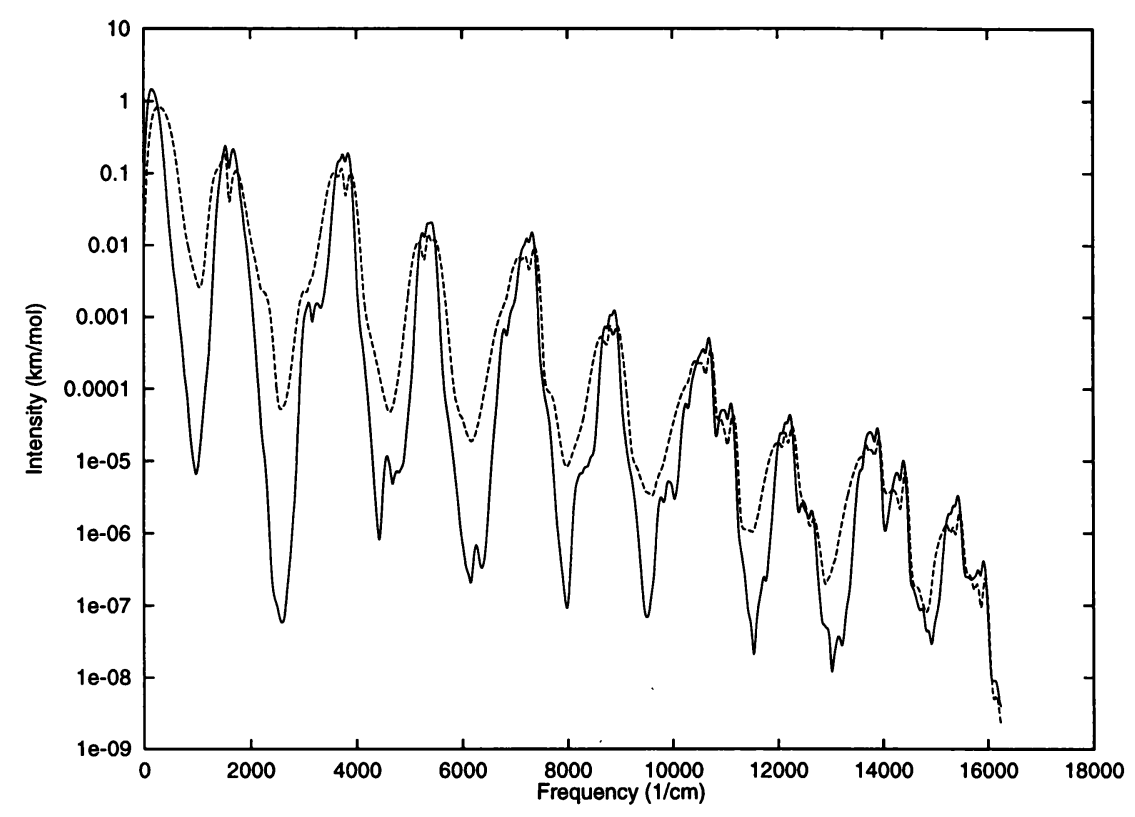

Figure 2. The spectra of $\mathrm{H}_{2} \mathrm{O}$ vapor at $296 \mathrm{~K}$ (solid line) and $1000 \mathrm{~K}$ (dashed line)

intensity of the individual lines is sensitive to the exact PES, the opacity distribution is much less so, and it is felt that the present calculations describe it quantitatively.

We are in the process of generating an opacity data base for high temperatures based on this new potential. The spectra at $296 \mathrm{~K}$ and at $1000 \mathrm{~K}$ based on this new potential are shown in Fig. 2. The most intense peak corresponds to pure rotational transitions in the (000) band, the second peak to transitions in the (010) band and so forth. At $296 \mathrm{~K}$ the spectra shows a characteristic band structure that can be associated with the different normal modes. As the temperature increases to $1000 \mathrm{~K}$, the valleys become less pronounced, but still the bands can be clearly differentiated. At still higher temperatures the structure is less apparent. We will be making our current opacity data base for $\mathrm{H}_{2} \mathrm{O}$ available very soon. Inquiries regarding the status and mechanism for acquiring the data base should be sent to David Schwenke (schwenke@pegasus.arc.nasa.gov). 


\section{References}

Brett, J. M. 1990, Astron. Astrophys., 231, 440

Carette, P., Schamps, J. 1992, J. Mol. Spectrosc., 154, 448

Collins, J. G. 1975, J. Phys. B 1975, 304, and references therein

Davis, S. P., Littleton, J. E., Phillips, J. G. 1986, ApJ, 309, 449

Doverstal, M., Weijnitz, P. 1992, Mol. Phys. 75, 1357

Dunning, T. H. 1989, J. Chem. Phys., 90, 1007

Feinberg, J., Davis, S. P. 1977, J. Mol. Spectrosc. 65, 264

Feinberg, J., Davis, S. P. 1978, J. Mol. Spectrosc. 69, 445

Hedgecock, I. M., Naulin, C., Costes M. 1995, Astron. Astrophys., 304, 667

Jørgensen, U. G. 1994, Astron. Astrophys., 284, 179

Jørgensen, U. G., Jensen, P. 1993, J. Mol. Spectrosc. 161, 219

Kendall, R. A., Dunning, T. H., Harrison, R. J. 1992, J. Chem. Phys., 96, 6796

Langhoff, S. R. 1997, Ap. J., submitted

Merrill, P. W., Deutsch, A. J., Keenan, P. C. 1962, Ap. J., 136, 21

Partridge, H., Schwenke, D. W. 1997, J. Chem. Phys. in preparation

Polyansky, O. L., Busler, J. R., Guo, B., Zhang, K., Bernath, P. F. 1996, J. Mol. Spectrosc. 176,305 , and references therein

Price, M. L., Sulzmann, K. G., Penner, S. S. 1974, J. Quant. Spectrosc. Radiat. Transfer, 14,1273

Rothman, L. S., Gamache, R., Schroeder, J. W., McCann, A., Wattson, R. B. 1995, SPIE Proc., 2471, 105

Schamps, J., Sennesal, J. M., Carette, P. J. 1992, J. Quant. Spectrosc. Radiat. Transfer, 48,147

Schwenke, D. W., 1996, J. Phys. Chem., 100, 2867

Simard, B., Hackett, P. A. 1991, J. Mol. Spectrosc., 148, 128

Steele, R. E., Linton, C. 1978, J. Mol. Spectrosc., 69, 66

Viti, S., Tennyson, J. 1997, Mon. Not. R. Astron. Soc., in press

\section{Discussion}

Martin: I am glad that you find higher values of the oscillator strengths for the $\mathrm{TiO} \delta$-bands than previous calculations show, because it helps to interpret the spectra of stars we have been studying, M-dwarfs mainly. Could you tell us by how much the oscillator strengths are larger? Is it by a factor of two?

Langhoff: Yes, almost exactly a factor of two larger than the current recommended values.

Zare: Might rotational mixing among different electronic states in $\mathrm{TiO}$ be improtant?

Langhoff: This is an interesting question. As you go to very high vibrational and rotational quantum numbers, the excited states are embedded in each others manifold and indeed a wave function could become a linear combination of different configurational characters, which could change the intensity of the transition. However, it is hoped that by summing over all levels of each excited electronic state, the correct opacity distribution will be obtained even though the details of the individual transitions may be deficient. Thus we believe that for opacity it will not be critical to account for this mixing. 